\section{Case Reports in Nephrology and Dialysis}

Case Rep Nephrol Dial 2020;10:154-162

\begin{tabular}{l|l}
\hline DOI: $10.1159 / 000510871$ & (C) 2020 The Author(s)
\end{tabular}

Published online: November 17, 2020

Published by S. Karger AG, Basel www.karger.com/cnd

This article is licensed under the Creative Commons Attribution-NonCommercial 4.0 International License (CC BY-NC) (http://www.karger.com/Services/OpenAccessLicense). Usage and distribution for commercial purposes requires written permission.

\title{
Fibrilo-Tactoid Glomerulonephritis: A Possible Novel Morphological Variant
}

\author{
Amaresh Vanga $^{a} \quad$ Sandeep Magoon ${ }^{a} \quad$ Jolanta Kowalewska ${ }^{b}$ \\ Saad Mussarat ${ }^{\mathrm{C}}$ \\ aDepartment of Nephrology and Hypertension, Eastern Virginia Medical School, \\ Norfolk, VA, USA; 'Department of Pathology, Eastern Virginia Medical School, \\ Norfolk, VA, USA; 'Department of Internal Medicine, Eastern Virginia Medical School, \\ Norfolk, VA, USA
}

\section{Keywords}

Glomerulonephritis · Proteinuria · Biopsy

\begin{abstract}
Fibrillary and immunotactoid glomerulonephritis are infrequent causes of primary nephrotic range proteinuria and are poorly understood. Recent significant developments include the discovery of DNA JB9 antigen in fibrillary glomerulonephritis. Here, we present a case of a middleaged woman who presented with nephrotic range proteinuria, hematuria, and normal renal function. Renal biopsy revealed fibrils that were randomly arranged on electron microscopy. They were of small size and congo red negative similar to the ones found in fibrillary glomerulonephritis, but were also DNA JB 9 negative, and had a hollow core like in immunotactoid glomerulopathy. Though we try to classify these conditions into either immunotactoid glomerulonephropathy (ITGN) or fibrillary glomerulonephritis (FGN), there are scenarios such as this case where it does not fit into either and is probably an overlap or intermediate variant of
\end{abstract}




\section{Case Reports in Nephrology and Dialysis}

Case Rep Nephrol Dial 2020;10:154-162

DOI: $10.1159 / 000510871$

Vanga et al.: Fibrilo-Tactoid Glomerulonephritis

these two conditions. Pathological features of these glomerulonephrites are discussed together with their clinical implications, treatment choices, and diagnostic importance.

(C) 2020 The Author(s)

Published by S. Karger AG, Basel

\section{Introduction}

Fibrillary and immunotactoid glomerulonephritis occurs in less than $1 \%$ of total kidney biopsies [1]; however, recent developments in this field, including DNA JB9 identification has tremendously increased our confidence in grouping them to one of either category. Morphological characteristics such as the diameter of fibrils, hollow nature of structures, arrangement of fibrils or tubules, along with other findings like immunoglobulin monoclonality, staining for DNAJB9, and identification of secondary diseases like paraproteinemias, infections such as hepatitis $\mathrm{C}$, autoimmune conditions, paraneoplastic syndromes, often aid in the classification of these fibrillary diseases. Here we present a case of organized IgG deposits that do not fit either of these variants despite the above resources and could be associated with unidentified antigen similar to DNA JB9.

\section{Case Presentation}

A 55-year-old female with a medical history significant for well-controlled diabetes mellitus complicated by proteinuria presented for a follow-up visit. On this visit, she reported shortness of breath, weight gain of $5.5 \mathrm{~kg}$, and lower extremity swelling. She denied any new over-the-counter medications usage, hemoptysis or hematuria, sore throat, rash, or fever. Her glycemic control was fair on oral hypoglycemics. Her outpatient medications included baby aspirin, losartan, metformin, zinc. Other past medical history included hypertension, morbid obesity, vitamin D deficiency, and history of tobacco abuse. She used to smoke one pack per day but quit a little over a year ago. The patient's proteinuria significantly changed, and microalbumin to creatinine ratio was $5.5 \mathrm{~g}$, which is considerably worse than her baseline of less than $500 \mathrm{mg}$.

Her vital signs were recorded as temperature of $36.8^{\circ} \mathrm{C}$, pulse $84 / \mathrm{min}$, blood pressure $138 / 78 \mathrm{~mm} \mathrm{Hg}$, respiratory rate 16/min. On examination, her chest was clear to auscultation, and no rubs, murmurs or gallops were noted. Pedal edema was present. Her labs were significant for microalbumin to creatinine ratio, which was elevated to 9,155 on repeat testing. Her microalbumin to creatinine ratio was less than 0.5 gms 4 months ago. Albumin dropped to 3.2 $\mathrm{mg} / \mathrm{dL}$, and it was within normal range 4 months ago. Her urine analysis showed proteinuria and blood (Table 1). Her creatinine was $0.9 \mathrm{mg} / \mathrm{dL}$ (at baseline) and Bun $17 \mathrm{mg} / \mathrm{dL}$. Due to the acute onset of nephrotic range proteinuria, kidney biopsy and serological workup were planned to find the exact etiology.

She was admitted to the hospital for CT-guided renal biopsy, and extensive serological workup was ordered. Her serum protein electrophoresis (SPEP) did not show monoclonal bands. She had a mild elevation of kappa light chains, but the kappa/lambda ratio came back as normal (1.23). She tested negative for HIV, cryoglobulins, hepatitis B and C. Her C3, C4, beta- 


\section{Case Reports in Nephrology and Dialysis}

Case Rep Nephrol Dial 2020;10:154-162

DOI: 10.1159/000510871

Vanga et al.: Fibrilo-Tactoid Glomerulonephritis

2-microglobulin were within the normal range (Table 2). Her antibodies, including antinuclear antibody (ANA), proteinase antibody (PR-3), myeloperoxidase antibody (MPO), glomerular basement membrane (GBM) antibody, were negative.

\section{Kidney Biopsy}

The renal biopsy sample was processed according to the standard techniques. Multiple sections were stained with hematoxylin and eosin (HE), periodic-acid Schiff's (PAS), John methenamine silver (JMS), and trichrome stain. The biopsy specimen showed the renal cortex containing 16-28 glomeruli per level section, of which one (5\%) was globally sclerosed. Approximately $50 \%$ of the glomeruli showed segmental mesangial and endocapillary hypercellularity due to cellular proliferation and influx of leukocytes, with many polymorphonuclear leukocytes (Fig. 1). At least two glomeruli showed small cellular crescents (Fig. 2) and at least two other showed segmental adhesions of glomerular tufts to the adjacent Bowman's capsules. The glomerular capillary walls were irregularly thickened with focal "spikes" of the basement membrane, segmental membrane vacuolization, or double focal contour (Fig. 3). Mild patchy interstitial fibrosis and tubular atrophy were noted (approximately 7\%). Occasional tubular segments contained red blood cells. Arteries and arterioles were without significant sclerosis or evidence of vasculitis. Staining with Congo red reagent for amyloid was negative.

Immunofluorescence microscopy showed granular, predominantly capillary wall staining for IgG(ab) and IgGw (both 1-2+), C3 (2+), kappa (trace to 1+). There was no significant glomerular staining for IgA, IgM, C1q, lambda light chains, fibrinogen, or albumin. There was no staining of the tubular basement membranes, interstitium, or vessels for any of the tested immune reactants. Analysis of IgG subclasses demonstrated a polyclonal staining pattern with antisera specific for IgG1 (2+), IgG2 (2+), and IgG4 (trace). Staining for IgG3 was negative.

Ultrastructural examination revealed numerous subepithelial and intramembranous immune type deposits. Many of the deposits were separated or surrounded by basement membrane-like-material. There was a frequent remodeling of capillary walls with focal duplication and cellular interposition. Occasional small mesangial and sub-endothelial deposits were also identified. The immune deposits were organized into randomly arranged, nonbranching fibrils measuring, on average $13.3 \mathrm{~nm}$ (range from 9.9 to $18.2 \mathrm{~nm}$ ) in thickness (Fig. 4a-d). The fibrils were somewhat rod-like, focally hollowed, and/or have cross-striations (with ladderlike appearance). The glomerular epithelial cells (podocytes) showed extensive effacement of foot processes. Many of the capillary loops contained circulating leukocytes, which focally encroached upon the capillary basement membrane causing significant thinning. Tubuloreticular inclusions were not seen in the endothelial or other cell types. No evidence of immune deposits was seen in the tubular basement membranes or the interstitium. Tubular cells contained lipid droplets.

Given the small fibrils size and negative staining for Congo red, the initial impression was fibrillary glomerulonephritis. However, additional staining for DNAJB9 (that is $98 \%$ sensitive and $>99 \%$ specific for the diagnosis of fibrillary glomerulonephritis) was found to be negative.

The final diagnosis was acute proliferative glomerulonephritis with an atypical pattern of organized IgG immunoglobulins. Bone marrow biopsy performed as a part of secondary workup following the results of kidney biopsy showed normocellular bone marrow with $40 \%$ cellularity with trilineage hematopoiesis and less than $5 \%$ blasts. No evidence of 


\section{Case Reports in Nephrology and Dialysis}

Case Rep Nephrol Dial 2020;10:154-162

DOI: 10.1159/000510871

Vanga et al.: Fibrilo-Tactoid Glomerulonephritis

myelodysplasia, lymphoproliferative disorder or plasma cell dyscrasia was noted. Fluorescence in situ hybridization (FISH) for multiple myeloma panel was found within normal limits. Cytogenetics did not suggest any abnormalities. Flow cytometry results did not suggest any definitive evidence of myeloid dysplasia, lymphoproliferative, or plasma cell dyscrasia. On clinical follow-up, the patient was initially treated with pulse steroids and subsequently received two doses of rituximab, $1 \mathrm{~g}$ each, 2 weeks apart. At follow-up, proteinuria dropped to the sub-nephrotic range with a resolution of edema and improvement in serum albumin. Her GFR has remained stable.

\section{Discussion/Conclusion}

Fibrillary and immunotactoid GN are extremely rare and need ultrastructural analysis for correct diagnosis. When associated with monoclonality, they are grouped under MGRS (monoclonal gammopathy of renal significance). MGRS classification is based on the presence of organized deposits and the size of fibrils [2]. Our patient had trace to 1+ kappa staining of deposits, but later subclassification of IGG on biopsy sample revealed a polyclonal IGG pattern, thereby excluding the monoclonality. This case also exemplifies the problem with making a definite pathological diagnosis based only on morphological classification in cases where there are micro-tubular or fibrillary structures in the deposits. Sometimes it is difficult for the pathologist to make this distinction as some of the fibrils may appear hollow from one end and can also be curved [1]. There has been a lot of debate in the past to appropriately classify immunotactoid and fibrillary GN, as some have argued that both represent the same disease but at different points in the spectrum $[3,4]$. Other investigators have also published articles identifying the various clinical, histological, and immunological patterns for these diseases and hence should not be considered as one [5-9]. Some of the patterns have an overlap of both disorders. Recent developments, such as DNA JB9 biomarker discovery, have further clarified these as distinct entities and help us in clearly distinguishing these two entities. As DNA JB9 is 98-100\% sensitive and close to $100 \%$ specific, the chances of having a false negative or positive are extremely rare and thought to be the hallmark of fibrillary GN [10]. This case is DNA JB9 negative, thereby arguing against fibrillary GN. However, it does not fit the typical picture of ITGN (immunotactoid glomerulonephropathy) either as there are randomly arranged fibrils of a mean thickness of $13.5 \mathrm{~nm}$ (range of 11-16.5) on electron microscopy, which is relatively small. Ultrastructural examination of the organized deposits revealed that some of the fibrils have a rod-like, focally hollow appearance and had an average thickness of $13.3 \mathrm{~nm}$ (range of 9.9-18.2). We can say that this represents an atypical case of ITGN but also argue that this morphology is somewhere in between ITGN and FGN, thereby raising the possibility again of a new morphological pattern of MGRS that could be coined as "fibril-tactoid GN." In an article published by Andeen et al. [11], a couple of cases were identified initially as proliferative GN with fibrillary deposits and later revised as probable immunotactoid GN based on negative DNA JB9 results. Both patients had a fibrillary diameter of 19-20 nm and had abnormal creatinine and eGFR, unlike the case that is presented here. Another differential diagnosis that can be considered in this scenario would be fibronectin glomerulopathy as this entity can be found in similar age groups with nephrotic presentation. However, there is scant staining 


\section{Case Reports in Nephrology and Dialysis}

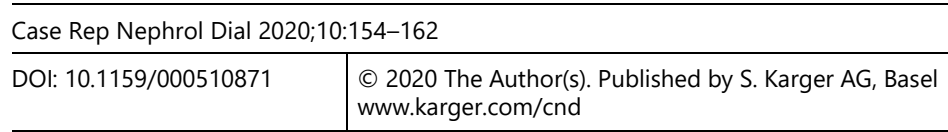

Vanga et al.: Fibrilo-Tactoid Glomerulonephritis

for immunoglobulins and complements in this scenario which was not the case in this patient [12].

It has been extensively reported in the literature regarding the importance of distinguishing ITGN from other types of Congo red negative fibrillary deposits due to its implication on the underlying cause, treatment, and overall prognosis. According to Nasr et al. [13], 38\% of cases with ITGN have an underlying lymphoproliferative disorder. Most such cases of ITGN have a concomitant or prior diagnosis of hematological malignancy like lymphoma. However, few case reports denote that a diagnosis of hematological malignancy was made months after the discovery of ITGN [14-16]. In a patient like the one we presented, high vigilance needs to be maintained for the future development of any malignancy. Although some would stress on morphological difference to distinguish fibrillary from immunotactoid GN, this can be arbitrary as the fibrils, in what has been called FGN and ITGN, range with considerable overlap from 10 to $49 \mathrm{~nm}[4,16]$. Some have argued that the morphologic definition of ITG also includes a "hollow," electron-lucent lumen in the fibrils, but morphologic studies have suggested that the fibrils in many, if not all, cases of what has been called fibrillary GN (FGN) also have an electron-lucent lumen $[18,19]$. This further ascertains the fact that morphological classification is more arbitrary as there is a significant overlap from the standpoint of fibril size and structure. Considerable weightage should be put on sensitive and specific markers, such as DNA JB9, to make the distinction between FGN and ITGN. However, in a case like ours, there is a possibility of unidentified antigens like DNA JB9 that could be teased out with mass spectroscopy. If we need to integrate the morphological classification along with these antigen statuses, then a term such as fibrilo-tactoid glomerulonephritis would be appropriate for such a case.

\section{Statement of Ethics}

All treatments and examinations followed the guidance of the Declaration of Helsinki. Informed consent for treatment and renal biopsy was obtained from the patient. The patient has given her written informed consent to publish the case, including the publication of images.

\section{Conflict of Interest Statement}

The authors have no conflicts of interest to declare

\section{Funding Sources}

This project was not supported by any grant or funding agencies. 


\section{Case Reports in Nephrology and Dialysis}

\begin{tabular}{l|l} 
Case Rep Nephrol Dial 2020;10:154-162 \\
\hline DOI: 10.1159/000510871 & $\begin{array}{l}\text { (c) 2020 The Author(s). Published by S. Karger AG, Basel } \\
\text { www.karger.com/cnd }\end{array}$
\end{tabular}

Vanga et al.: Fibrilo-Tactoid Glomerulonephritis

\section{Author Contributions}

Amaresh Vanga and Saad Mussarat contributed to the acquisition of data and writing of the manuscript. Jolanta Kowalewska provided renal biopsy imaging and interpretation of renal biopsy pathology, and review. Sandeep Magoon provided supervision, assessment, interpretation of data along with mentorship. All authors approved the final manuscript.

\section{References}

1 Alpers CE, Kowalewska J. Fibrillary glomerulonephritis and immunotactoid glomerulopathy. J Am Soc Nephrol. 2008 Jan;19(1):34-7.

2 Bridoux F, Leung N, Hutchison CA, Touchard G, Sethi S, Fermand JP, et al.; International Kidney and Monoclonal Gammopathy Research Group. Diagnosis of monoclonal gammopathy of renal significance. Kidney Int. 2015 Apr;87(4):698-711.

3 Brady HR, Cohen JJ, Harrington JT, Madias NE, Zusman CJ. Fibrillary glomerulopathy. Kidney Int. 1998 May;53(5):1421-9.

4 Schwartz MM, Korbet SM, Lewis EJ. Immunotactoid glomerulopathy. J Am Soc Nephrol. 2002 May;13(5):1390-7.

5 Alpers CE. Immunotactoid (microtubular) glomerulopathy: an entity distinct from fibrillary glomerulonephritis? Am J Kidney Dis. 1992 Feb;19(2):185-91.

6 Fogo A, Qureshi N, Horn RG. Morphologic and clinical features of fibrillary glomerulonephritis versus immunotactoid glomerulopathy. Am J Kidney Dis. 1993 Sep;22(3):367-77.

7 Rosenstock JL, Markowitz GS, Valeri AM, Sacchi G, Appel GB, D’Agati VD. Fibrillary and immunotactoid glomerulonephritis: distinct entities with different clinical and pathologic features. Kidney Int. 2003 Apr;63(4):1450-61.

8 Alpers CE. Fibrillary glomerulonephritis and immunotactoid glomerulopathy: two entities, not one. Am J Kidney Dis. 1993 Sep;22(3):448-51.

9 Bridoux F, Hugue V, Coldefy O, Goujon JM, Bauwens M, Sechet A, et al. Fibrillary glomerulonephritis and immunotactoid (microtubular) glomerulopathy are associated with distinct immunologic features. Kidney Int. 2002 Nov;62(5):1764-75.

10 Nasr SH, Vrana JA, Dasari S, Bridoux F, Fidler ME, Kaaki S, et al. DNAJB9 is a specific immunohistochemical marker for fibrillary Glomerulonephritis. Kidney Int Rep. 2017 Aug;3(1):56-64.

11 Andeen NK, Troxell ML, Riazy M, Avasare RS, Lapasia J, Jefferson JA, et al. Fibrillary Glomerulonephritis: Clinicopathologic Features and Atypical Cases from a Multi-Institutional Cohort. Clin J Am Soc Nephrol. 2019 Dec;14(12):1741-50.

12 Ishimoto I, Sohara E, Ito E, Okado T, Rai T, Uchida S. Fibronectin glomerulopathy. Clin Kidney J. 2013 Oct;6(5):513-5.

13 Nasr SH, Fidler ME, Cornell LD, Leung N, Cosio FG, Sheikh SS, et al. Immunotactoid glomerulopathy: clinicopathologic and proteomic study. Nephrol Dial Transplant. 2012 Nov;27(11):4137-46.

14 Khandelwal A, Trinkaus MA, Ghaffar H, Jothy S, Goldstein MB. A case report of unusually long lag time between immunotactoid glomerulopathy (itg) diagnosis and diffuse large B-cell lymphoma (DLBCL) development. BMC Nephrol. 2016 Sep;17(1):140.

15 Vigil A, Oliet A, Gallar P, Ortega O, Rodriguez Villarreal I, Picazo L, et al. Rapidly progressive immunotactoid glomerulonephritis and multiple myeloma. Nephron. 1998;79(2):238-40.

16 Jacobson E, Sharp G, Rimmer J, MacPherson B. A 59-year-old woman with immunotactoid glomerulopathy, heavy-chain disease, and non-hodgkin lymphoma. Arch Pathol Lab Med. 2004 Jun;128(6):689-92.

17 Korbet SM, Schwartz MM, Lewis EJ. Immunotactoid glomerulopathy. Am J Kidney Dis. 1991 Mar;17(3): 247-57.

18 Korbet SM, Schwartz MM, Rosenberg BF, Sibley RK, Lewis EJ. Immunotactoid glomerulopathy. Medicine (Baltimore). 1985 Jul;64(4):228-43.

19 Korbet SM, Schwartz MM, Lewis EJ. The fibrillary glomerulopathies. Am J Kidney Dis. 1994 May;23(5): 751-65. 


\section{Case Reports in Nephrology and Dialysis}

Case Rep Nephrol Dial 2020;10:154-162

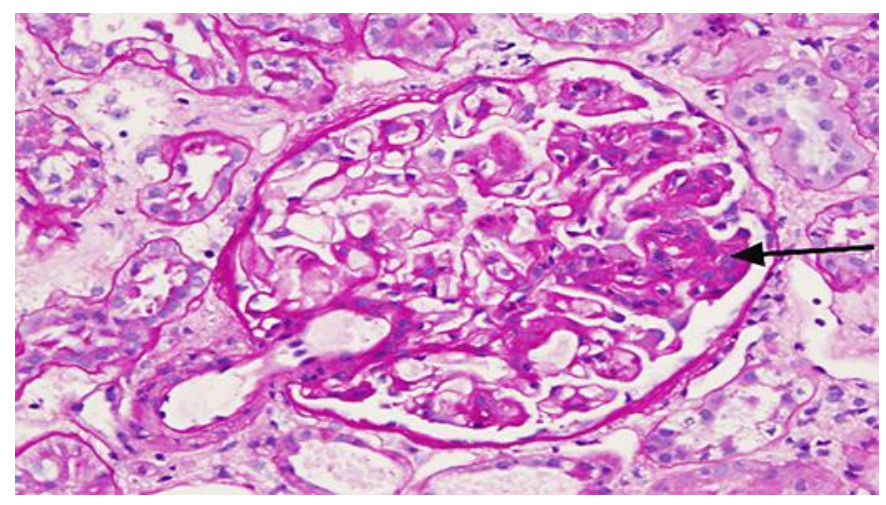

Fig. 1. Glomerulus with segmental mesangial and endocapillary hypercellularity with influx of leukocytes. PAS, original magnification $400 \times$.

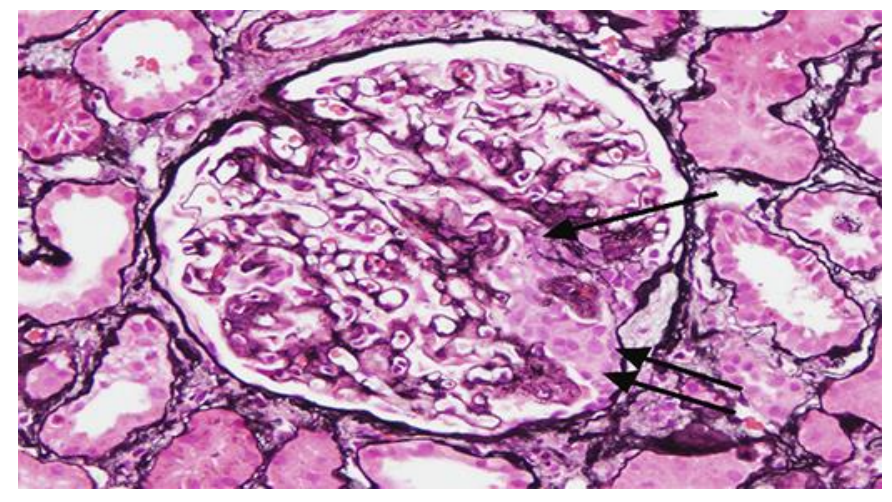

Fig. 2. Glomerulus with focal break in basement membrane (arrow) and a small cellular crescent (double arrow). JMS, original magnification 400x. 


\section{Case Reports in Nephrology and Dialysis}

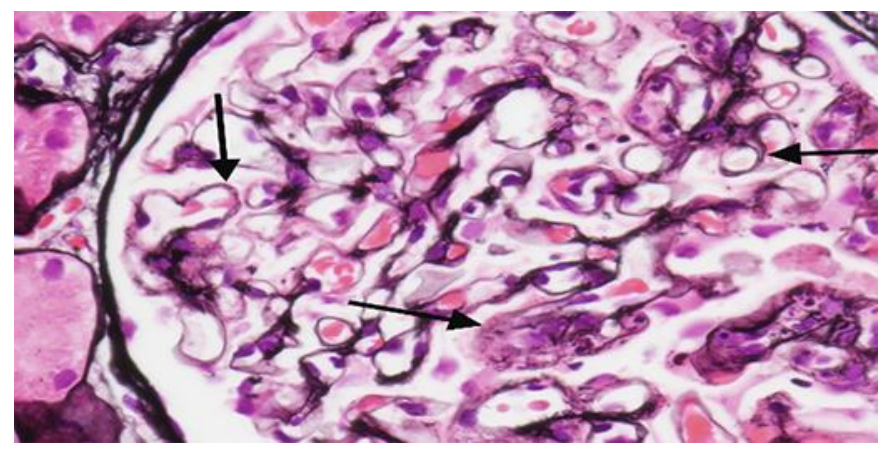

Fig. 3. Irregular appearance of glomerular capillary walls. JMS, original magnification 600×.
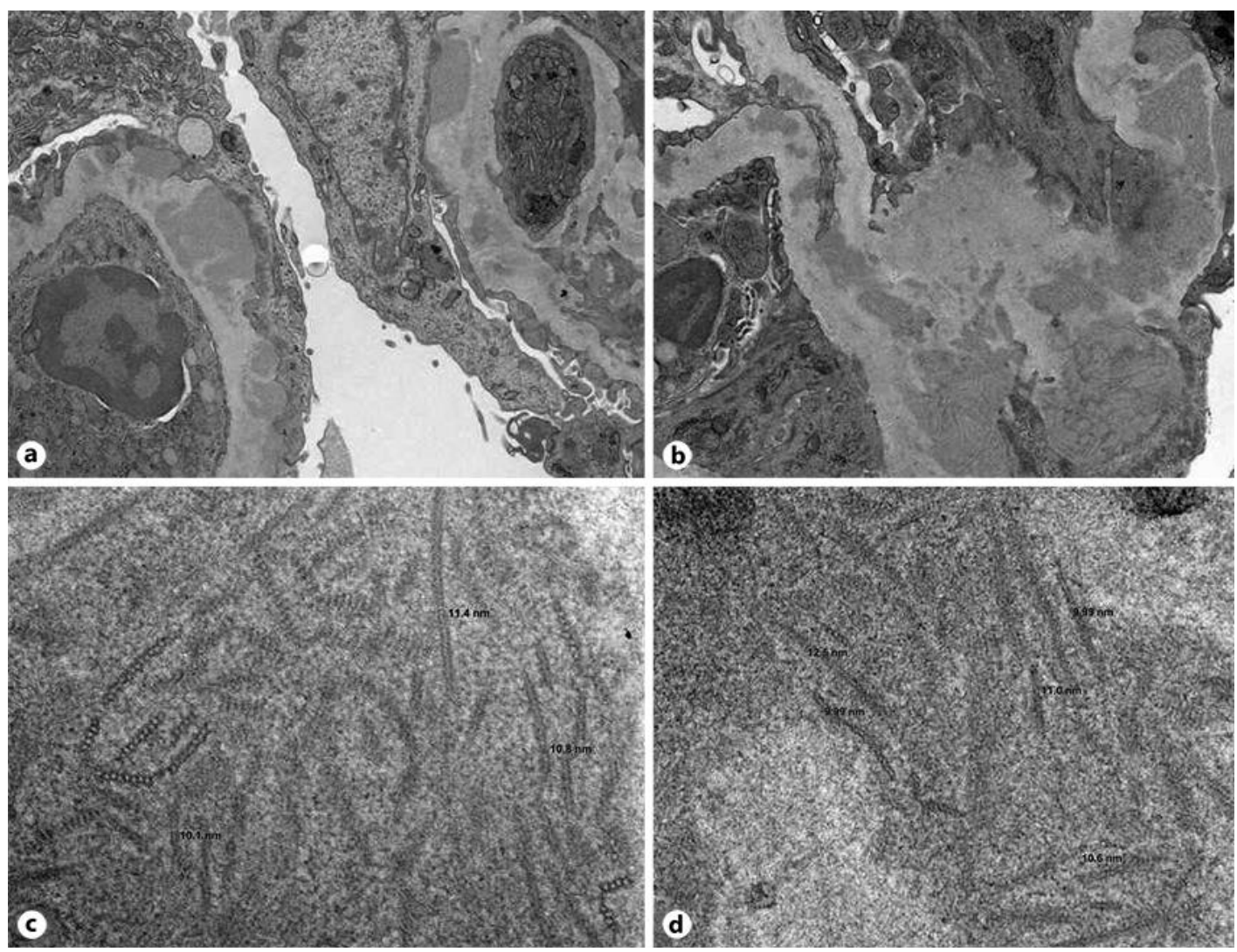

Fig. 4. a Organized immune deposits in the subepithelial region. Direct magnification 2,500×. b Organized immune deposits in the subepithelial and intramural region. Direct magnification 2,500x. c Fibrils with hollow, rod like and ladder like appearance. Direct magnification 30,000x. d Fibrils randomly arranged with hollow, rod like appearance. Direct magnification 25,000×. 


\section{Case Reports in Nephrology and Dialysis}

Table 1. Urinalysis

\begin{tabular}{lll}
\hline Assay & Value & Reference range \\
\hline Urine specific gravity & 1.015 & $1.005-1.030$ \\
Urine pH & 6 & $5-8$ \\
Urine protein screen & $>500$ & Negative, trace mg/dL \\
Urine glucose & Negative & Negative \\
Urine blood & Large & Negative \\
Urine nitrite & Negative & Negative \\
Urine leukocyte esterase & Small & Negative \\
Urine WBC & $50-100$ & $0-2 / \mathrm{hpf}$ \\
Urine RBC & Loaded & Negative \\
\hline
\end{tabular}

Table 2. Key laboratory studies

\begin{tabular}{lll}
\hline Assay & Values & Reference range \\
\hline Hemoglobin, g/dl & 13.5 & $11.7-16$ \\
White blood cell count, k/ $\mu \mathrm{L}$ & 7.6 & $4.0-11.0$ \\
Platelet count, k/ $\mu \mathrm{l}$ & 283 & $140-440$ \\
Blood urea nitrogen, mg/dL & 17 & $6-22$ \\
Creatinine, mg/dL & 0.9 & $0.5-1.2$ \\
Cryoglobulins & undetected & $\mathrm{NA}$ \\
SPEP, IFE & No suspicious monoclonal look- & $\mathrm{NA}$ \\
& ing bands seen & \\
HIV, HBV PCR, HCV RNA PCR & Negative & $\mathrm{NA}$ \\
C3, mg/dl & 150 & $83-177$ \\
C4, mg/dl & 27 & $10-40$ \\
ANA, units & Negative & NA \\
PR3-ANCA,units & $<3.5$ & $0-3.5 \mathrm{U} / \mathrm{mL}$ \\
MPO-ANCA, units & $<9$ & $0-9 \mathrm{U} / \mathrm{mL}$ \\
Anti-GBM, units & 3 & $0-20 \mathrm{units}$ \\
Beta 2 microglobulin & 1.9 & $0.6-2.4 \mathrm{mg} / \mathrm{L}$ \\
\hline
\end{tabular}

SPEP, serum protein electrophoresis; IFE, immunofixation electrophoresis; HIV, human immunodeficiency virus; HBV, hepatitis B; PCR, polymerase chain reaction, hepatitis C; C3, C3 complement; C4, C4 complement; ANA, antinuclear antibody; ANCA, perinuclear antineutrophil cytoplasmic antibodies; MPO, myeloperoxidase; PR3, proteinase 3; GBM, glomerular basement membrane. Note: Conversion factors for units: serum creatinine in $\mathrm{mg} / \mathrm{dL}$ to $\mu \mathrm{mol} / \mathrm{L}, \times 88.4$; serum urea nitrogen in $\mathrm{mg} / \mathrm{dL}$ to $\mathrm{mmol} / \mathrm{L}, \times 0.35$. 